\title{
Anxiety linked to symptom severity in bipolar disorder
}

Published online: 23 October 2012

(C) Springer Healthcare 2012

medwireNews: Anxiety is common in bipolar disorder patients experiencing an episode of acute mania and is associated with increased mood symptom severity, research shows.

Researchers found that almost half of bipolar disorder patients with acute mania had moderate to severe anxiety levels, and higher levels of anxiety were associated with greater levels of mania and depression.

"Further studies are necessary to determine whether effective treatment of anxiety symptoms could lessen bipolar disorder severity or improve response to treatment of bipolar symptoms," comment Ana González-Pinto (University of the Basque Country, Vitoria, Spain) and team.

The findings, published in Acta Psychiatrica Scandinavica, come from a study of 242 patients ( $57 \%$ women), aged an average of 43 years, with bipolar disorder who were admitted to hospital with an acute episode of mania. All of the patients had a score of at least 20 on the Young Mania Rating Scale (YMRS).

The patients were assessed for anxiety within 24 hours of admission using the Hamilton Anxiety Rating Scale (HARS). This is a 14-item test of anxiety severity, with each item scored on a 5point scale ranging from o (not present) to 4 (severe).

The researchers found that the patients' mean HARS score was 14.3 , with $46 \%$ meeting criteria for moderate to severe anxiety, with a HARS score greater than 14.0.

In multiple linear regression analysis, higher HARS scores were significantly associated with increased severity of manic and depressive symptoms, as measured using the YMRS and the Clinical Global Impression for Bipolar Disorder scale, respectively.

Furthermore, patients with moderate to severe anxiety had a 20\% longer hospital stay than those with HARS scores of 14 or less, at a mean of 21 days versus 18 days, respectively.

González-Pinto and team conclude: "The findings of this study corroborate the close relationship between anxiety and manic symptoms in patients with acute mania, highlighting the need for greater clinical attention to anxiety in this population.”

By Mark Cowen, Senior medwireNews Reporter

\section{Reference}

Acta Psychiatr Scand 2012; 126: 351-355 